\title{
LETTER FROM THE EDITOR IN CHIEF
}

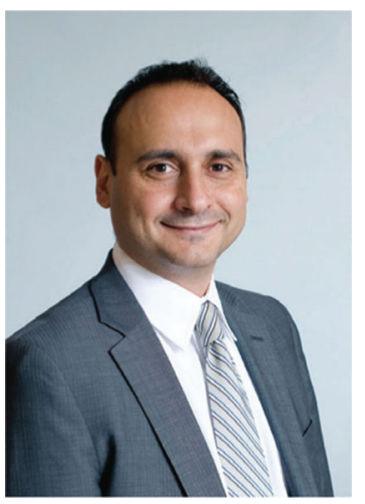
congenital heart disease. Also this study has some limitations, the most important of which is the design being a retrospective single center study. Despite the limitations, this study is important because it demonstrated that a negative EP study in congenital heart disease patients suggests a low risk for sudden cardiac death, which in my opinion has important clinical implications.

Another reason the study is important is because it highlights the risk of sudden cardiac death in patients with congenital heart disease. Because of medical and technological advances, the vast majority of children with congenital heart disease are surviving into adulthood. This is a large population and comprises over 1 million people in the US. Cardiac rhythm disorders are one of the most challenging complications among these patients. The congenital structural abnormalities and the scars from subsequent surgeries result in reentry tachycardias in the atria and ventricles, some of which are life-threatening. Clinical studies aiming at finding techniques and technologies to predict and prevent sudden cardiac death in this population are of critical importance.

Another important article in this issue is the elegant review by John Day summarizing the most impactful clinical studies in 2015. These included the role of weight loss in reducing the AF burden, leadless pacing, and the low yield of performing DFTs. These landmark publications will likely affect the clinical practice.

I hope you enjoy reading this issue of the Journal.

Regards,

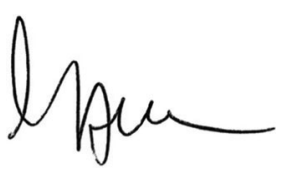

Moussa Mansour, MD, FHRS, FACC

Editor-in-Chief

The Journal of Innovations in Cardiac Rhythm Management

MMansour@InnovationsInCRM.com

Director, Cardiac Electrophysiology Laboratory

Director, Atrial Fibrillation Program

Massachusetts General Hospital Boston, MA 